\title{
Flavonoid intake and disability-adjusted life years due to Alzheimer's and related dementias: a population-based study involving twenty-three developed countries
}

\author{
Kristopher Beking and Amandio Vieira* \\ Nutrition \& Metabolic Research Laboratory, Biomedical Physiology \& Kinesiology, and Institute for Health \\ Research \& Education, Simon Fraser University, Burnaby, British Columbia, V5A 1S6, Canada
}

Submitted 26 February 2009: Accepted 11 November 2009: First published online 11 January 2010

\begin{abstract}
Objective: Dietary flavonoids and their metabolites may have neuroprotective effects against age-associated neurodegenerative disorders such as Alzheimer's and related dementias (dementia). There is a lack of population studies, however, on correlations between flavonoid intake and dementia. The main objective of the present study was to analyse such a relationship at a large-scale population level. Design: Based on global data (FAO, WHO), databases were generated for: (i) flavonoid content of foods; (ii) per capita national dietary intakes of flavonoids and other dietary factors; and (iii) disability-adjusted life years - a measure of burden and death - due to dementia. Five major flavonoid subclasses were examined. To minimize influences due to accuracy and reliability of the disease source data, twenty-three developed countries were selected after statistical evaluation.

Results: Flavonols and combined flavonoids (all five combined) intakes were the only two parameters with significant $(P<0.05)$ negative dementia correlations. Multiple linear regression models confirmed this relationship, and excluded confounding from some other dietary and non-dietary factors. Similar analyses with non-dementia, neurological/psychiatric diseases did not yield significant correlations. Conclusions: At a global level, and in the context of different genetic backgrounds, our results suggest that higher consumption of dietary flavonoids, especially flavonols, is associated with lower population rates of dementia in these countries.
\end{abstract}

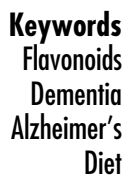

Keywords

Flavonoids

Alzheimer's

Diet
There are numerous reports on biochemical mechanisms that may contribute to the neuroprotective effects of flavonoids, especially in the context of Alzheimer's and related, age-associated neurodegenerative disorders ${ }^{(1-5)}$. In terms of epidemiological evidence, a prospective cohort study has reported a risk ratio of 0.49 for dementia between the highest and lowest tertiles of flavonoid intake $^{(6)}$; and another study indicated a protective relationship between flavonoid intake and risk of dementia only among smokers ${ }^{(7)}$. More recent studies on the Mediterranean diet - a diet typically rich in flavonoids from fruits and vegetables and wine - have associated adherence to this diet with lower risk of developing Alzheimer's and mild cognitive impairment, and conversion of such impairment to Alzheimer's ${ }^{(8-10)}$. Moreover, regular consumption of flavonoid-rich foods such as tea and wine has been associated with better performance on cognitive tests and decreased risk of cognitive decline in elderly populations in Asia and Europe ${ }^{(11,12)}$. Our present study provides (to our knowledge) the first test for an inverse ecological association between dietary intakes of flavonoids and global-level rates of Alzheimer's and other related dementias (hereafter called 'dementia') among a large number of developed countries in different continents.

An epidemiological study, the European National Variation in Burden of Disease and Nutrition ${ }^{(13)}$, as well as The World Health Report ${ }^{(14)}$, served as conceptual resources for our study. Our methodology is comparable to other global ecological studies that relate nutrition to disease; for example, a 2006 study $^{(15)}$ compared diabetes incidence in various world regions with dietary parameters from the FAO Food Balance Sheets ${ }^{(16)}$. Social variables that may have an influence on dementia rates were also considered in our study: educational attainment of the population ${ }^{(17)}$, ethnic distribution ${ }^{(18)}$, socioeconomic conditions $^{(19)}$ and gender distributions of the population over 65 years of age ${ }^{(20)}$.

The disability-adjusted life year (DALY) was used as the parameter for the diseases examined in the current study. A DALY is a measure of the burden that a disease has on those afflicted in a population. It represents a mathematical combination of years of life lost prematurely due to the disease and years of life spent in disability, normally 


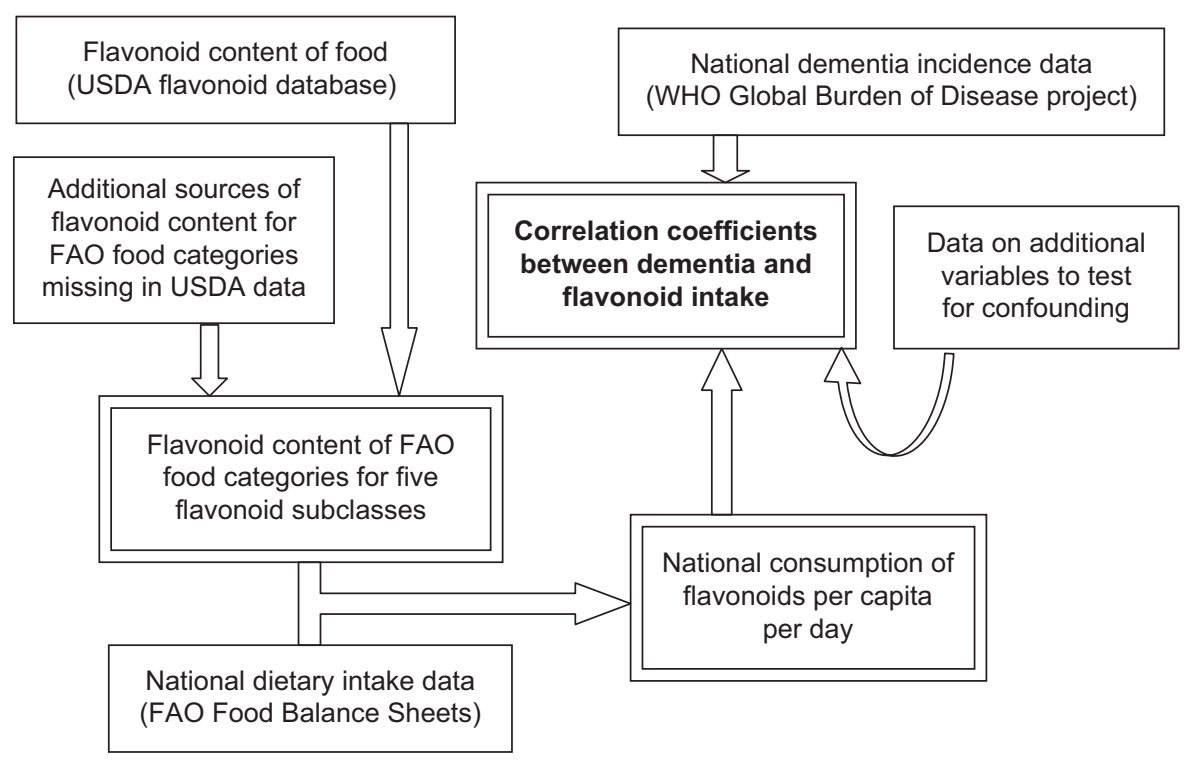

Fig. 1 Flowchart of methods - database production and data analyses. Single-lined boxes indicate data from external sources; double-lined boxes indicate processed data; bold text indicates analyses (USDA, US Department of Agriculture)

given as a rate (e.g. per 100000$)$. With the 2006 publication of the vast and ongoing Global Burden of Disease (GBD) project $^{(21)}$ came extensive disease burden statistics, including data for 'Alzheimer's and other senile dementias ${ }^{,(22)}$.

\section{Experimental methods}

A schematic flowchart of the methods - database production and analysis steps - is shown in Fig. 1. Database preparation and statistical analyses are further detailed below.

\section{Disease databases}

Global statistics for the incidence of dementia were obtained from the WHO Burden of Disease Statistics ${ }^{(22)}$. Accuracy and reliability of the disease data source were subject to the following considerations.

In relation to socio-economic environment, the WHO describes data as they pertain to distinct world regions: a high-income region, representative of the developed world, and several other geographically based regions, representative of the developing world. Health information coverage was $99 \%$ for the former, but typically much lower for the latter groups ${ }^{(21)}$. This disparity in coverage is consistent with a previous estimation of world dementia rates that found data sources for developing nations either to be incomplete or to have diseasereporting formats inconsistent with the developed world, or non-existent altogether ${ }^{(23,24)}$. The GBD study assigned to each country a rating for each of three levels representative of different aspects of data reliability (rating of 1 , most reliable). If differences within these levels between countries influence dementia rates, then including data from multiple nations with inconsistent levels could render the results less accurate by introducing a possible confounding factor in the source data. The first two levels each had four possible ratings, 1 (best) to 4 (worst); the third had only two possible ratings ( 3 or 4). Analyses of variance (more details below) were used to determine whether the level of evidence of a country was an influencing factor on dementia rates. Income status was also tested as a source of variance in the dementia data and additionally as an interacting factor with the levels of evidence ratings. Twenty-three countries (list below) were selected such that the ratings for each level of evidence would not, upon interaction with income status or alone, significantly act as confounding factors on the dementia rates.

Economics and health-care funding data were also collected to enable testing for any residual confounding influence ${ }^{(25)}$ apart from that controlled by nation selection (see below); these included gross national income and total expenditure on health care. To minimize ethnic influences from possibly confounding flavonoid-based effects on correlations, predominantly Caucasian nations were selected as units of study: European countries (Austria, Belgium, Denmark, Finland, France, Germany, Greece, Iceland, Ireland, Italy, Luxembourg, Netherlands, Norway, Portugal, Slovenia, Spain, Sweden, Switzerland, UK), New Zealand, Australia, USA and Canada; it is apparent from the database ${ }^{(22)}$, and supported by the globally based dementia prevalence studies ${ }^{(23,24,26)}$, that rates of dementia are affected by the primary ethnicity of a country.

The disease data set used in the current study had already been adjusted for variations in age distributions ${ }^{(25)}$. Data on gender were collected as male:female ratio in the population over 65 years of age ${ }^{(27)}$. Data on education were collected as percentage of the population enrolled in 
Table 1 National age-standardized disability-adjusted life year rates of dementias in relation to level of evidence and country income

(a) Levelst of evidence and national income status as factors

\begin{tabular}{lrl}
\hline Factor & \multicolumn{1}{c}{$F$} & $P$ value \\
\hline Level 1 & 83.895 & $<0.001^{*}$ \\
Level 2 & 52.049 & $<0.001^{*}$ \\
Level 3 & 115.065 & $<0.001^{*}$ \\
Income & 124.954 & $<0.001^{*}$
\end{tabular}

(b) Levels of evidence as primary factors, interacting with income status as secondary factors

\begin{tabular}{|c|c|c|c|c|c|}
\hline Factor 1 & Factor 2 & $F$ (level) & $F$ (income) & $F$ (interaction) $\ddagger$ & $P$ value $\S$ \\
\hline Level 1 & Income & $44 \cdot 124$ & $18 \cdot 86$ & $3 \cdot 688$ & $0.027^{\star}$ \\
\hline Level 2 & Income & $7 \cdot 524$ & $56 \cdot 492$ & 1.082 & $0 \cdot 341$ \\
\hline Level 3 & Income & $13 \cdot 723$ & $46 \cdot 988$ & 1.095 & 0.297 \\
\hline
\end{tabular}

(c) Levels of evidence as factors, high-income countries only

\begin{tabular}{lcc}
\hline Factor & $F$ & $P$ value \\
\hline Level 1 & 3.773 & $0.033^{\star}$ \\
Level 2 & 1.095 & 0.346 \\
Level 3 & 0.765 & 0.388
\end{tabular}

${ }^{*} P<0.05$.

tLevels are distinct rating systems given by WHO representing quality of dementia data. fInteraction between level and income (high $v$. rest), ANOVA.

$\S P$ value represents statistical significance of the interaction.

tertiary schooling ${ }^{(28)}$. As a statistical control for disease, using the same methods as with dementia, data ${ }^{(22)}$ on a group of neuropsychiatric conditions from GBD statistics, excluding Alzheimer's and related dementias, were also collected.

\section{Dietary factors databases}

Data on dietary consumption were obtained from FAO Food Balance Sheets ${ }^{(16)}$ in the form of grams per capita per day (g/cap per d) for flavonoid-containing foods (105 categories of grains, roots and tubers, sugar products, legumes, nuts, seed oils, fruits, vegetables, wine, beer, tea and spices). The FAO's collection methods are comprehensive, all-inclusive, not sample-based, and are robust and reliable for use in national- or world region-based nutrition studies. The determination of flavonoid content for each of these categories involved the use of information from the US Department of Agriculture (USDA) on the flavonoid content of selected foods ${ }^{(29)}$. Because some categories of minor grains and spices were not covered in the USDA publication, additional literature sources on food flavonoid content were used ${ }^{(30-44)}$. The result was the creation of two flavonoid databases, one with USDAonly data as a source and one with USDA data supplemented with additional sources; the latter was used for all relevant figures and tables in the present study. For each of the food categories, the calculated $\mathrm{mg} / 100 \mathrm{~g}$ value of each of the flavonoid subclasses and combined flavonoids (sum of all five subclasses) was listed. This database was then cross-tabulated with dietary consumption data for each country to form a new data set for each country containing the $\mathrm{mg} / \mathrm{cap}$ per $\mathrm{d}$ values on daily consumption of each flavonoid subclass, and of combined flavonoids.
Correlation coefficients were calculated between data sets of flavonoid consumption and DALY rates of dementia for each country (see Fig. 1). As a non-flavonoid control, values for antioxidant vitamins ${ }^{(16,45)}$ were also used to calculate correlation coefficients.

\section{Statistical analyses}

Analyses of variance were carried out using the SPSS statistical software package version 12 (SPSS Inc., Chicago, IL, USA), with rates of dementia as the dependent variable. The $\alpha$ value for statistical significance was $0 \cdot 05$. In the first round, ANOVA was tested for each level of evidence as well as income status, among all nations given in the source data (Table 1a). In the second round (Table 1b), factorial ANOVA was used to test whether income status had an interaction with each of the three levels in their influence on dementia rates. This could indicate if the variance seen in Table $1 \mathrm{a}$ is due to income status. ANOVA was used again in the third round (Table 1c) to test for variance of dementia for the three levels of evidence for high-income countries only. After controlling for highincome status, ethnicity and availability of Food Balance Sheets, all remaining nations had either a first or second rating for each level of evidence. Three subsequent rounds of $t$ tests were performed to test whether dementia rates varied significantly between the first and second ratings (these two differed in completeness and year of data) for each of the levels of evidence. Then $t$ tests were used to determine whether dementia rates among highincome countries would vary depending on which of the top two ratings for each level of evidence was used (Table 2a; 4th round), and then repeated with a control for ethnicity (Table $2 \mathrm{~b}$; 5 th round); a final repetition was 
Table 2 National age-standardized disability-adjusted life year rates of dementias in relation to level of evidence, income and ethnicity

(a) Comparison of first two ratingst for each levelł, high-income countries only

\begin{tabular}{lll}
\hline Factor & $t$ Test & $P$ value \\
\hline Level 1 & $2 \cdot 746$ & $0.010^{\star}$ \\
Level 2 & 1.25 & 0.22 \\
Level 3 & 0.875 & 0.388
\end{tabular}

(b) Comparison of first two ratings for each level, high-income countries only and controlled for ethnicity

\begin{tabular}{lcc}
\hline Factor & $t$ Test & $P$ value \\
\hline Level 1 & 6.205 & $<0.001^{*}$ \\
Level 2 & 0.637 & 0.571
\end{tabular}

(c) Comparison of first two ratings for each level, all countries

\begin{tabular}{lrr}
\hline Factor & $t$ Test & $P$ value \\
\hline Level 1 & $6 \cdot 117$ & $<0.001^{*}$ \\
Level 2 & $3 \cdot 195$ & $0.002^{*}$ \\
Level 3 & $10 \cdot 727$ & $<0.001^{*}$ \\
\hline
\end{tabular}

${ }^{\star} P<0.05$.

tRatings are the measure of quality of data for the given level. łLevels are defined in Table 1 .

done (Table 2c; 6th round) for all nations, to test whether the variance remained constant across levels (as with all nations in Table 1).

Stepwise multiple regression analysis was performed with five flavonoid subclasses as the independent variables and rates of dementia as the dependent variable, to test which ones significantly contribute to dementia while adjusting for each other. Further multiple regression analyses were performed with rates of dementia as the dependent variable and combined flavonoid intake as the independent variable, and each of the other factors (education, sex, gross national income, total expenditure on health, intake of vitamin A, C and E) entered individually as the other independent variable. Factors that showed significant influence on dementia rates, alongside the flavonoid variable, were considered possible confounding factors, as were factors that showed significant correlation coefficients with both a flavonoid variable and dementia rates in the correlation matrices.

\section{Results}

Population rates of dementia varied significantly depending on the rating for each level of evidence, and even more so depending on income status (Table 1a). A significant interaction between levels of evidence and income status occurred only for the first level (Table 1b). When controlling for income status by selecting only high-income nations, variance within level 1 significantly affected dementia rates, while variance within levels 2 and 3 did not (Table 1c). Hence, controlling for income
Table 3 Correlation coefficients between national flavonoid per capita intake and disability-adjusted life year rates of dementias

\begin{tabular}{lccc}
\hline Flavonoid category & $r$ & $r^{2}$ & $P$ value \\
\hline Anthocyanidins & -0.304 & 0.093 & 0.158 \\
Flavanols & -0.309 & 0.095 & 0.152 \\
Flavanones & -0.299 & 0.089 & 0.166 \\
Flavones & -0.164 & 0.027 & 0.455 \\
Flavonols & -0.436 & 0.19 & $0.038^{*}$ \\
Combined flavonoids & -0.416 & 0.173 & $0.048^{*}$ \\
\hline
\end{tabular}

${ }^{\star} P<0.05$.

prevents confounding from the rating for levels 2 and 3; and possible confounding by the rating among highincome countries should be controlled for level 1 .

Among high-income nations, dementia variance was dependent on which of the first two ratings was used for the first level of evidence (Table 2a). For the second and third levels, using nations of the first two ratings did not affect dementia rate variation. This means that only the first rating of level 1 should be used to prevent confounding, but using the first two ratings for levels 2 and 3 will not confound the dementia rates. Data from highincome countries only have first or second ratings for levels 2 and 3; hence, control for high income also offers a measure of quality assurance of the data. These effects were maintained when controlling for ethnicity (Table 2b); hence, the nations selected (level 1, rating 1; level 2, all ratings) for the current study will not be biased from their ratings for each level of evidence. When including all nations, the dementia rates varied significantly between the first two ratings for each of the three levels (Table 2c), in agreement with Table 1a.

Correlation coefficients between age-adjusted DALY for dementia and the flavonoid subclasses and combined flavonoid intake (Table 3) showed negative correlations ( $r$ ) for each of the six flavonoid classes and two-tailed statistical significance $(P<0.05)$ for two of them. One of the flavonoid subclasses, flavonols, had a higher correlation coefficient than total flavonoids. The dementia variable had an average value of 228 (SD 18.9) DALY/100 000 people. Correlations with USDA-only data (see Experimental methods; data not shown) were similar for all flavonoids to those in Table 3: flavonols, $r=-0 \cdot 435, P=0 \cdot 038$. Proanthocyanidins, polymers of flavonols with relatively low intestinal absorption $^{(46)}$, were not extensively analysed in the current study; their correlation with dementia - obtained after supplementing the database ${ }^{(47)}$ - was not statistically significant: $r=-0 \cdot 317, \quad P=0 \cdot 141$. Isoflavones were not examined, as they were not part of USDA source data ${ }^{(29)}$.

None of the correlation coefficients of flavonoids with a control group of neurological/psychiatric disorders was statistically significant (Table 4). This disease group includes combined data on all neuropsychiatric disorders from the WHO source data with the exception of Alzheimer's and related dementias ${ }^{(22)}$. In the context of potentially neuroprotective antioxidant activities, correlations between 
antioxidant vitamins $\mathrm{A}, \mathrm{C}$ and $\mathrm{E}$ and rates of dementia were determined (Table 5); none of these was statistically significant. Consumption of fruits and vegetables (g/cap per d) was also not significantly correlated with dementia (Table 5). Table 6 shows the correlations between all six flavonoid categories, as well as dementia, with variables that may influence dementia rates (see Discussion); none of the variables showed significant correlations with either flavonoids or dementia.

In a stepwise multiple linear regression model with the five flavonoid subclasses as independent variables and dementia as the dependent variable, flavonols was the only variable that remained. In further models that had dementia as the dependent variable, and flavonols and each of the additional variables significantly correlated with flavonols $(P<0 \cdot 5$; alcohol consumption, vitamins $\mathrm{A}+\mathrm{C}+\mathrm{E}$ com-

Table 4 Correlation coefficients between national flavonoid per capita intake and a group of neurological/psychiatric disorderst that excludes Alzheimer's and related dementias

\begin{tabular}{lrrr}
\hline Flavonoid category & \multicolumn{1}{c}{$r$} & \multicolumn{1}{c}{$r^{2}$} & $P$ value \\
\hline Anthocyanidins & 0.013 & $<0.001$ & 0.953 \\
Flavanols & 0.262 & 0.069 & 0.226 \\
Flavanones & -0.014 & $<0.001$ & 0.948 \\
Flavones & -0.208 & 0.043 & 0.34 \\
Flavonols & -0.036 & 0.001 & 0.87 \\
Combined flavonoids & 0.074 & 0.005 & 0.736 \\
\hline
\end{tabular}

tDisorders include bipolar and unipolar depressive, schizophrenia, epilepsy, alcohol abuse, Parkinson's, multiple sclerosis, drug abuse, post-traumatic stress, obsessive-compulsive, panic, insomnia and migraine.

Table 5 Correlations between national per capita intake of antioxidant vitamins, or total fruits and vegetables, and national disability-adjusted life year rates of dementias

\begin{tabular}{lccc}
\hline Nutrient category & $r$ & $r^{2}$ & $P$ value \\
\hline Vitamin A & 0.056 & 0.003 & 0.799 \\
Vitamin C & -0.335 & 0.112 & 0.118 \\
Vitamin E & -0.037 & 0.001 & 0.867 \\
Vitamins A + C + E & -0.069 & 0.005 & 0.755 \\
Fruits/vegetables & -0.275 & 0.076 & 0.204 \\
\hline
\end{tabular}

bined, anthocyanins, flavones) entered individually as independent variables, only flavonols remained in each model. Similarly, in models that had dementia as the dependent variable, and combined flavonoids and each of the other factors individually as independent variables, only flavonoids remained in each model. Thus, none of these variables acted as confounders in correlations of flavonols or flavonoids with dementia rates. As a means of adjusting for two other variables commonly adjusted in dementia studies $^{(17-20)}$, a further multiple linear regression was performed using the enter method (Table 7 ): the standardized correlation coefficient $(\beta)$ for total flavonoids remained significant and was higher, -0.546 ; the other two variables remained insignificant (Table 7 ).

\section{Discussion}

The present study provides evidence for a significant negative correlation between intake of some flavonoids and DALY of dementia at a large-scale population level. Such correlations were not found for a group of control neurological and psychiatric diseases that excludes dementias. Intake of all five flavonoid subclasses was negatively correlated with dementia DALY; of these five, only flavonols had a robust, statistically significant negative correlation with dementia incidence. The correlation of combined flavonoid intake and dementia rates was also negative and statistically significant; the $r^{2}$ value (coefficient of determination; Table 3) suggests that

Table 7 Gender, education and national flavonoid per capita intake $(\mathrm{mg} / \mathrm{d})$ as predictorst of disability-adjusted life year rates of dementias

\begin{tabular}{lcc}
\hline $\begin{array}{l}\text { Variables included in } \\
\text { model }\end{array}$ & $\begin{array}{l}\text { Standardized } \\
\text { coefficient } \beta\end{array}$ & $P$ value \\
\hline Combined flavonoid intake & -0.546 & $0.011^{\star}$ \\
Ratio of males:females & -0.201 & 0.333 \\
Population in tertiary schooling & -0.337 & 0.102 \\
\hline${ }^{*} P<0.05$. & & \\
tBased on multiple regression analyses. & &
\end{tabular}

Table 6 Correlations of national flavonoid per capita intakes or disability-adjusted life year rates of dementia, with potentially confounding variables

\begin{tabular}{|c|c|c|c|c|c|c|c|}
\hline & Dementia & Anthocyanidins & Flavanols & Flavanones & Flavones & Flavonols & Flavonoids \\
\hline \multicolumn{8}{|c|}{ Male:female ratio for age 65 years +} \\
\hline$r$ & $-0 \cdot 141$ & -0.392 & -0.254 & 0.009 & $-0 \cdot 124$ & $-0 \cdot 188$ & -0.310 \\
\hline$P$ value & 0.520 & 0.064 & 0.243 & 0.967 & 0.573 & 0.389 & $0 \cdot 150$ \\
\hline \multicolumn{8}{|c|}{ Total expenditure on health care } \\
\hline$r$ & $-0 \cdot 130$ & -0.066 & 0.536 & $0 \cdot 185$ & -0.040 & -0.209 & $0 \cdot 172$ \\
\hline$P$ value & 0.556 & 0.766 & $0.008^{*}$ & 0.397 & 0.855 & 0.339 & 0.433 \\
\hline \multicolumn{8}{|c|}{ Gross national income } \\
\hline$r$ & -0.079 & $0 \cdot 218$ & 0.400 & $0 \cdot 381$ & 0.099 & $-0 \cdot 163$ & 0.353 \\
\hline$P$ value & 0.720 & 0.318 & 0.059 & 0.072 & 0.654 & 0.457 & 0.098 \\
\hline \multicolumn{8}{|c|}{ Percentage of population in tertiary schooling } \\
\hline$r$ & -0.293 & -0.261 & -0.231 & 0.042 & $-0 \cdot 159$ & 0.002 & -0.202 \\
\hline$P$ value & 0.176 & 0.229 & 0.288 & $0 \cdot 848$ & 0.470 & 0.993 & 0.356 \\
\hline
\end{tabular}


combined flavonoid intake may account for about $17 \%$ of the variation in dementia rates. It should be noted, however, that such analyses have limitations; for example, flavonoids may be merely markers of healthy foods, and the resulting potential neuroprotection may be a result of particular food combinations (cf. Table 5 discussion below). In both the USDA-only (see Experimental methods) and supplemented-USDA (cf. Table 3) databases, flavonol intake was more strongly correlated with decreased dementia DALY than intake of total flavonoids.

Specific features of flavonoids and their metabolites may account for potentially neuroprotective activities ${ }^{(1-5)}$ in relation to dementia. Flavonols are often the most potent antioxidants in different oxidation assays, such as those based on hydrogen donation and metal chelation, and this has been related to several properties distinct to their structure $^{(48)}$. These properties, in combination with others such as bioavailability, redox cycling with other antioxidants, and metabolic activation, may contribute to potential neuroprotection. Differences between flavonols and other flavonoids/dietary factors in terms of synergism of antioxidant properties with other physiological activities - modulation of signal transduction, apoptosis, proteolysis, metabolic enzyme activity, membrane integrity, interaction with amyloidogenic proteins, and others ${ }^{(4,5,48-54)}$ - may also be important in this context.

Of the antioxidant nutrient controls (vitamins A, C and $\mathrm{E}$ and $\mathrm{A}+\mathrm{C}+\mathrm{E}$ combined) only vitamin $\mathrm{C}$ intake showed a notable, though statistically insignificant, negative correlation. In a neuroprotection context, other studies have also suggested a greater potency of flavonoids relative to vitamin $C^{(5,47,55)}$.

Dietary flavonoids derive predominantly from the consumption of fruits and vegetables ${ }^{(13)}$. The relatively weaker correlation between total fruit and vegetable intake and rates of dementia (Table 5) suggests that those fruits and vegetables which contain the most flavonoids, especially flavonols, are more strongly correlated with dementia than fruits and vegetables in general, and that flavonoids may be the component responsible for most of the potential neuroprotective effects. Our results emphasize the flavonoid components of foods, and are comparable to earlier studies of flavonoids and other dietary factors and risk of $\mathrm{CHD}^{(56,57)}$. Additional factors that could be accounted for (gender ratio, income, education) did not appear to have a confounding effect. Thus, based on a multinational study of developed countries in different continents, we provide evidence for a significant inverse correlation between dietary consumption of flavonoids, especially flavonols, and DALY rates of dementia.

\section{Acknowledgements}

This work was supported by a Natural Sciences and Engineering Research Council of Canada (NSERC) Discovery
Grant (A.V.) and a Canadian Institutes of Health Research (CIHR) Graduate Student Scholarship (K.B.). The authors report no conflicts of interest. Author contributions were as follows. A.V.: study design, orchestration, preparation of manuscript; K.B.: study design, statistical analyses, preparation of manuscript. We thank Dr P. Vieira (Nutrition and Metabolic Research Laboratory, Simon Fraser University) for helpful comments on this manuscript.

\section{References}

1. Ji HF \& Zhang HY (2006) Theoretical evaluation of flavonoids as multipotent agents to combat Alzheimer's disease. J Mol Struct 767, 3-9.

2. Youdim KA \& Joseph JA (2001) A possible emerging role of phytochemicals in improving age-related neurological dysfunctions: a multiplicity of effects. Free Radic Biol Med 30, 583-594.

3. Hirohata M, Hasegawa K, Tsutsumi-Yasuhara S et al. (2007) The anti-amyloidogenic effect is exerted against Alzheimer's $\beta$-amyloid fibrils in vitro by preferential and reversible binding of flavonoids to the amyloid fibril structure. Biochemistry 46, 1888-1889.

4. Oteiza PI, Erlejman AG, Verstraeten SV et al. (2005) Flavonoid-membrane interactions: a protective role of flavonoids at the membrane surface? Clin Dev Immunol 12, 19-25.

5. Williams RJ, Spencer JPE \& Rice-Evans C (2004) Flavonoids: antioxidants or signalling molecules? Free Radic Biol Med 36, 838-849.

6. Commenges D, Scotet V, Renaud S et al. (2000) Intake of flavonoids and risk of dementia. Eur J Epidemiol 16, 357-363.

7. Engelhardt MJ, Geerlings MI, Ruitenberg A et al. (2000) Dietary intake of antioxidants and risk of Alzheimer's disease. JAMA 287, 3223-3229.

8. Scarmeas N, Stern Y, Mayeux R et al. (2009) Mediterranean diet and mild cognitive impairment. Arch Neurol 66, 216-225.

9. Scarmeas N, Stern Y, Mayeux R et al. (2006) Mediterranean diet, Alzheimer disease, and vascular mediation. Arch Neurol 63, 1709-1717.

10. Scarmeas N, Stern Y, Tang MX et al. (2006) Mediterranean diet and risk for Alzheimer's disease. Ann Neurol 59, 912-921.

11. Ng TP, Feng L, Niti M et al. (2008) Tea consumption and cognitive impairment and decline in older Chinese adults. Am J Clin Nutr 88, 224-231.

12. Nurk E, Refsum H, Drevon CA et al. (2009) Intake of flavonoid-rich wine, tea, and chocolate by elderly men and women is associated with better cognitive test performance. J Nutr 139, 120-127.

13. Pomerleau J, McKee M, Lobstein T et al. (2003) The burden of disease attributable to nutrition in Europe. Public Health Nutr 6, 453-461.

14. World Health Organization (2002) The World Health Report. Geneva: WHO.

15. Muntoni S \& Muntoni S (2006) Epidemiological association between some dietary habits and the increasing incidence of type 1 diabetes worldwide. Ann Nutr Metab 50, 11-19.

16. Food and Agriculture Organization of the United Nations (2007) Food Balance Sheets. http://faostat.fao.org/site/555/ default.aspx, http://faostat.fao.org/site/502/default.aspx (accessed October 2007).

17. McDowell I, Xi G, Lindsay J et al. (2007) Mapping the connections between education and dementia. J Clin Exp Neuropsychol 29, 127-141. 
18. Tang MX, Cross P, Andrews H et al. (2001) Incidence of AD in African-Americans, Caribbean Hispanics, and Caucasians in northern Manhattan. Neurology 56, 49-56.

19. Goldbourt U, Schnaider-Beeri M \& Davidson M (2007) Socioeconomic status in relationship to death of vascular disease and late-life dementia. J Neurol Sci 257, 177-181.

20. Perneczky R, Diehl-Schmid J, Forstl H et al. (2007) Male gender is associated with greater cerebral hypometabolism in frontotemporal dementia: evidence for sex-related cognitive reserve. Int J Geriatr Psychiatry 22, 1135-1140.

21. Lopez AD, Mathers CD, Ezzati M et al. (editors) (2006) Global Burden of Disease and Risk Factors. Washington, DC/New York, NY: The World Bank/Oxford University Press.

22. World Health Organization (2006) Death and DALY estimates for 2002 by cause for WHO member states, Burden of Disease Statistics. http://www.who.int/ healthinfo/bod/en/index.html (accessed September 2006).

23. Wimo A, Winblad B, Aguero-Torres H et al. (2003) The magnitude of dementia occurrence in the world. Alzheimer Dis Assoc Disord 17, 63-67.

24. Ferri CP, Prince M, Brayne C et al. (2005) Global prevalence of dementia: a Delphi consensus study. Lancet 366 , 2112-2117

25. World Health Organization (2006) Core Health Indicators from World Health Statistics, WHO Statistical Information System (WHOSIS). The World Health Report, 2006 Edition. http://www3.who.int/whosis/core/core_select.cfm (accessed March 2007).

26. Fratiglioni L, De Ronchi D \& Aguero-Torres H (1999) Worldwide prevalence and incidence of dementia. Drugs Aging 15, 365-375.

27. Central Intelligence Agency (2007) Field Listing - Sex Ratio. The World Factbook. https://www.cia.gov/library/publications/ the-world-factbook/fields/2018.html (accessed October 2007).

28. The World Bank Group (2002) Educational attainment in the adult population. Barro-Lee Data Set, EdStats. http:// devdata.worldbank.org/edstats/query/default.htm (accessed May 2007)

29. Nutrient Data Laboratory, Agricultural Research Service, US Department of Agriculture (2007) USDA Database for the Flavonoid Content of Selected Foods, Release 2.1. Beltsville, MD: USDA.

30. Harnly JM, Doherty RF, Beecher GR et al. (2006) Flavonoid content of US fruits, vegetables, and nuts. J Agric Food Chem 54, 9966-9977.

31. Gu L, House SE, Wu X et al. (2006) Procyanidin and catechin contents and antioxidant capacity of cocoa and chocolate products. J Agric Food Chem 54, 4057-4061.

32. Miller KB, Stuart DA, Smith NL et al. (2006) Antioxidant activity and polyphenol and procyanidin contents of selected commercially available cocoa-containing and chocolate products in the United States. J Agric Food Chem 54, 4062-4068.

33. Wu X, Beecher GR, Holden JM et al. (2006) Concentrations of anthocyanidins in common foods in the United States and estimations of normal consumption. J Agric Food Chem 54, 4069-4075.

34. Peterson J, Dwyer J, Bhagwat S et al. (2005) Major flavonoids in dry tea. J Food Compost Anal 18, 487-501.

35. Auger C, Al-Awwadi N, Bornet A et al. (2004) Catechins and procyanidins in Mediterranean diets. Food Res Int 37, 233-245.

36. Arts ICW, Hollman PC \& Kromhout D (1999) Chocolate as a source of tea flavonoids. Lancet 354, 488.

37. Arts ICW (1999) Chocolate contains additional flavonoids not found in tea. Lancet 354, 1825.

38. Adom KK \& Liu RH (2006) Antioxidant activity of grains. J Agric Food Chem 50, 6182-6187.
39. Adom KK, Sorrells ME \& Liu RH (2005) Phytochemicals and antioxidant activity of milled fractions of different wheat varieties. J Agric Food Chem 53, 2297-2306.

40. Kamath VG, Chandrashekar A \& Rajini PS (2004) Antiradical properties of sorghum (Sorghum bicolor L. Moench) flour extracts. J Cereal Sci 40, 283-288.

41. Holtekjolen AK, Kinitz C \& Knutsen SH (2006) Flavanol and bound phenolic acid contents in different barley varieties. J Agric Food Chem 54, 2253-2260.

42. Shan B, Cai YZ, Sun M et al. (2005) Antioxidant capacity of 26 spice extracts and characterization of their phenolic constituents. J Agric Food Chem 53, 7749-7759.

43. The Wine Institute (2007) Per capita wine consumption in listed countries, 2002. Trade Data and Analysis. http:// www.wineinstitute.org/industry/keyfacts/docs/PerCapita WineConsumptionCountries.pdf (accessed October 2007).

44. Kirin Holdings Company, Ltd. (2004) Per capita beer consumption. http://www.kirinholdings.co.jp/english/ir/ news_release051215_4.html (accessed October 2007).

45. Nutrient Data Laboratory, Agricultural Research Service, US Department of Agriculture (2007) USDA National Nutrient Database for Standard Reference, Release 20. Beltsville, MD: USDA; available at http://www.nal.usda.gov/fnic/ foodcomp/search/index.html

46. Nutrient Data Laboratory, Agricultural Research Service, US Department of Agriculture (2004) USDA Database for the Proanthocyanidin Content of Selected Foods. Beltsville, MD: USDA.

47. Lotito SB \& Frei B (2006) Consumption of flavonoid-rich foods and increased plasma antioxidant capacity in humans: cause, consequence, or epiphenomenon? Free Radic Biol Med 41, 1727-1746.

48. Heim KE, Tagliaferro AR \& Bobilya DJ (2002) Flavonoid antioxidants: chemistry, metabolism and structure-activity relationships. J Nutr Biochem 13, 572-584.

49. Agullo G, Gamet-Payrastre L, Manenti S et al. (1997) Relationship between flavonoid structure and inhibition of phosphatidylinositol 3-kinase: a comparison with tyrosine kinase and protein kinase C inhibition. Biochem Pharmacol 53, 1649-1657.

50. Chen D, Daniel KG, Chen MS et al. (2005) Dietary flavonoids as proteasome inhibitors and apoptosis inducers in human leukemia cells. Biochem Pharmacol 69, 1421-1432.

51. Shimmyo Y, Kihara T, Akaike A et al. (2008) Flavonols and flavones as BACE-1 inhibitors: structure-activity relationship in cell-free, cell-based and in silico studies reveal novel pharmacophore features. Biochim Biophys Acta 1780, 819-825.

52. Padilla E, Ruiz E, Redondo S et al. (2005) Relationship between vasodilation capacity and phenolic content of Spanish wines. Eur J Pharmacol 517, 84-91.

53. Kalkbrenner F, Wurm G \& von Bruchhausen F (1992) In vitro inhibition and stimulation of purified prostaglandin endoperoxide synthase by flavonoids: structure-activity relationship. Pharmacology 44, 1-12.

54. Bastianetto S, Brouillette J \& Quirion R (2007) Neuroprotective effects of natural products: interaction with intracellular kinases, amyloid peptides and a possible role for transthyretin. Neurochem Res 32, 1720-1725.

55. Yao Y \& Vieira A (2007) Protective activities of Vaccinium antioxidants with potential relevance to mitochondrial dysfunction and neurotoxicity. Neurotoxicolgy 28, 93-100.

56. Hertog MG, Feskens EJ, Hollman PC et al. (1993) Dietary antioxidant flavonoids and risk of coronary heart disease: the Zutphen Elderly Study. Lancet 342, 1007-1011.

57. Hertog MG, Feskens EJM \& Kromhout D (1997) Antioxidant flavonols and coronary heart disease risk. Lancet 349, 699. 